\title{
Carrier Scattering Mechanisms in Bismuth Films Doped with Tellurium
}

\author{
D.Yu. Matveev* \\ Astrakhan State University, 20a, Tatischev st., 4140056 Astrakhan, Russia
}

(Received 15 May 2016; published online 03 October 2016)

\begin{abstract}
The article compares carrier mobility in monocrystals, as well as monocrystal and block films of different width thus defining carrier contribution to interaction with phonons, surface, boundaries and structural defects of crystallites in bismuth films doped with tellurium. It is determined that there is a linear dependence of inverse electron mobility on inverse width of bismuth film doped with tellurium.
\end{abstract}

Keywords: Thin films, Bismuth, Tellurium, Mobility, Size effect.

DOI: $10.21272 /$ jnep.8(3).03012

PACS numbers: 73.50.Gr, 73.50.Jt

\section{INTRODUCTION}

At present an overwhelming majority of the researches was dedicated to searching for the optimal conditions to obtain bismuth films doped with tellurium and provide for their high level of structural perfection and studying the influence of structure on physical properties of bismuth films [1]. The work on researching the doping influence on the structure and the galvanomagnetic phenomena in thin bismuth films has mainly been carried out abroad; it remains sporadic and lacks system [2-7].

For example, Orlova and Rogacheva's research results revealed that the use of thermal evaporation of bismuth crystals doped with tellurium in vacuum allows for deeper doping of bismuth films than bulk bismuth crystals [2]. Heremans and Dilner [3-6] note, that an increase of tellurium admixture concentration leads to expansion of low field area and to a significant decrease of electron mobility.

Based on the Mayadas-Shatzkes model in FuchsSondheimer theory, the authors [7] connect this fact with the crystallite boundaries making a significant contribution to electron scattering in block bismuth films doped with tellurium.

In this connection there arose the necessity to obtain monocrystallic bismuth films doped with tellurium in attempt to exclude crystallite boundaries contribution to carrier mobility limitations, thus approaching closely tridimensional monocrystal properties.

The article aims to define the influence of size (film width), doping degree and structural defects on carrier mobility in block and monocrystallic bismuth films doped with tellurium based on the determined regular change patterns of galvanomagnetic coefficients.

\section{METHODS OF THE EXPERIMENT}

The block bismuth films doped with tellurium were obtained by means of discrete thermal evaporation on the muscovite mica substrate in vacuum under pressure of $2 \cdot 10^{-3} \mathrm{~Pa}$. The discrete method was employed due to significant difference between pressures of bismuth and tellurium vapors at evaporation temperature of bismuth. Muscovite mica was chosen as a substrate material as mica has an orienting effect on crystalline structure of evaporated bismuth films. As a result, threefold $\mathrm{C}_{3}$ axis is perpendicular to the substrate plane in the obtained films, and it considerably facilitates the analysis and interpretation of the experimental data $[1,8]$

To improve crystalline structure, deposition of bismuth films doped with tellurium achieved by means of vacuum evaporation was carried out with optimal technological parameters to obtain pure bismuth films [1]. A bismuth crystal with adequate concentration of tellurium admixture was used as a source material.

Time-of-flight mass spectrometry performed by LUMAS-30 spectrometer with low-pressure pulsed glow discharge in a combined hollow cathode was employed to control the contents of tellurium in the original crystal [9]. Samples to analyze quantitative composition - thin plates, $10 \times 10 \mathrm{~mm}$ in size, $1 \mathrm{~mm}$ in width, were cut out from the middle of original crystal and fixed as a bottom of hollow cathode in gas-discharge cell, where pulsed ionization of sample atoms occurred in glow-discharge plasma. It was supposed the tellurium concentration in the film and monocrystal did not change.

Monocrystal bismuth films doped with tellurium were obtained by means of floating-zone refining under coating to differentiate the influence of film surface and crystal boundaries on the carrier scattering in these films $[8,10]$.

The width of all films was defined by means of multibeam optical interferometry with MII-4 microscope. The width of monocrystal film was deemed equal to the width of block film.

To study all the obtained films, Solver scanning probe microscope produced by NT-MDT company was used employing the method of atomic force microscopy in semi-contact regime and DRON-7, X-ray diffractometer, was applied to implement the rotating crystal method with the scheme of $\Theta-2 \Theta$ scanning in copper anode radiation $[1,8]$.

The measurements of the galvanomagnetic effects for all the obtained films were carried out by means of the automated device. The measurements of the specific electrical resistivity $\rho_{11}$, the relative transverse magnetoresistance $\rho_{1133}$ and Hall coefficient $R_{12,3}$ were car-

*Danila200586@mail.ru 
ried out in steady-state conditions following classical methodology. Relative error of the specific resistivity measurement was approximately $12 \%$, Hall constant approximately $14 \%$, magnetoresistance - approximately $5 \%$.

The results of the measurements and their description are considered in detail in papers [8]. We are not going to dwell on them in this paper, as it concerns processing of experimental data.

\section{SCATTERING MECHANISMS IN BLOCK AND MONOCRYSTAL BISMUTH TELLURIDE FILMS}

\subsection{Calculation of the Carrier Mobility in Bis- muth Films with 0.15 at. $\%$ and 0.075 at. $\%$ Te Contents}

It is known that the galvanomagnetic coefficient changes in the bismuth films doped with tellurium are fully defined by the temperature dependencies of concentration and carrier mobility.

In this connection the easiest option is to calculate electron mobility in the interval under study for films with 0.150 at.\% Te and films with 0.075 at. \% Te within temperature range of 77-100 $\mathrm{K}$ employing the component expression $\rho_{11}$ and taking into consideration that only electrons are carriers under the given tellurium concentration and the $u_{2}^{-}<<u_{1}^{-}$correlation of electron mobilities in trigonal plane [8].

In this case expression for transport coefficients $\rho_{11}$, $\rho_{1133}$ and $R_{12,3}$ are simplified:

$$
\begin{gathered}
1 / \rho_{11}=0,5 \operatorname{en}\left(u_{1}^{-}+u_{2}^{-}\right) \\
R_{12,3}=-e \rho_{11}^{2} n u_{1}^{-} u_{2}^{-} \\
\rho_{11,33}=0,5 \rho_{11}^{2} e n u_{1}^{-} u_{2}^{-}\left(u_{1}^{-}+u_{2}^{-}\right)-R_{12,3}^{2} / \rho_{11}
\end{gathered}
$$

where $n$ is the concentration of electrons, $e$ is the electron charge.

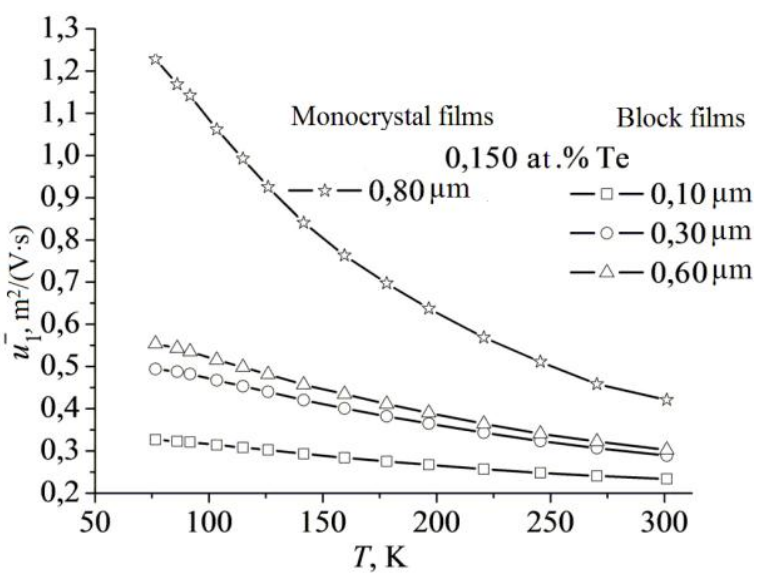

Fig. 1 - The temperature dependencies of electron mobility in the block and monocrystal bismuth films doped with tellurium; tellurium contents is 0.150 at. \%; films are of different width

According to the data for the bulk Bi crystals with
0.150 at. $\%$ Te, the electron concentration is equal to $n=2.95 \cdot 10^{25} \mathrm{~m}^{-3}$ [11]. For bulk Bi crystals with 0.075 at. $\% \mathrm{Te}$ the electron concentration is equal to $n=1.42 \cdot 10^{25} \mathrm{~m}^{-3}$ [11]. The temperature dependencies of electron mobility $\mathrm{u}_{1}{ }^{-}$for the $\mathrm{Bi}$ films with 0.075 at. \% Te and 0.150 at. \% Te and different width are shown in Fig. 1-2.

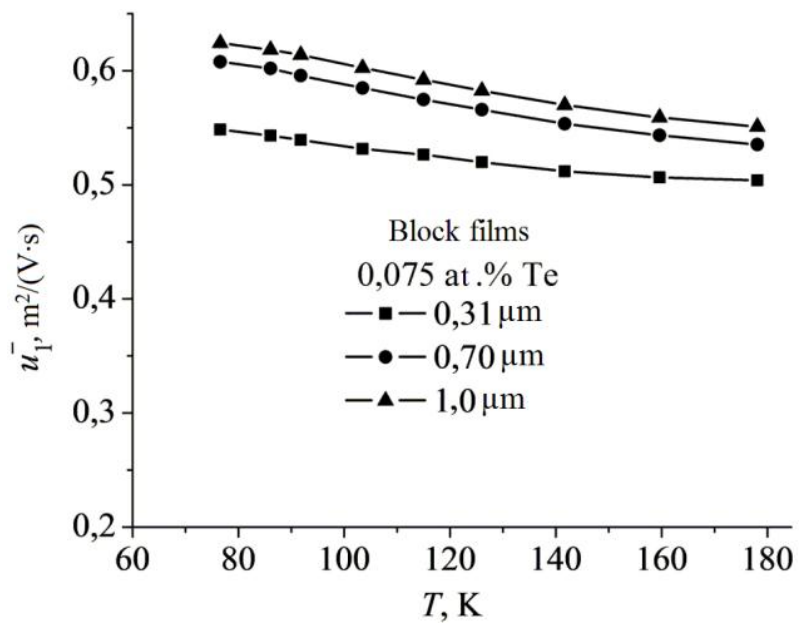

Fig. 2 - The temperature dependencies of electron mobility in the block and monocrystal bismuth films doped with tellurium; tellurium contents is 0.075 at. \%; films are of different width

Figures 1-2 show that in all the films under study the electron mobility $u_{1}^{-}$increases with temperature decease. The decrease in width is accompanied by the decrease in electron mobility that is caused by classic size effect [8].

The mobility of the monocrystal $\mathrm{Bi}$ film with 0.150 at. \% Te contents is higher than that of the block films with the same contents throughout the temperature range.

\subsection{Calculation of the Carrier Mobility in the Bismuth Films with 0.05 at. \% Te Contents}

Let us carry out the calculation of carrier mobility for the Bi films (0.05 at.\% Te).

It is notable that absolute value of Hall coefficient [8] of the Bi films (0.05 at.\% Te) considerably decreases when the width of the film gets smaller in $77-110 \mathrm{~K}$ temperature range, this decrease is usually associated with the rising carriers concentration [2-6]. However, taking into consideration that $u_{2}^{-}<<u_{1}^{-}$[8] in bismuth crystals, expressions (1) and (2) imply that the observed dependence of Hall coefficient on film width can also be caused by changes in the ratio of carrier mobility components [8]

$$
R=-4 u_{2}^{-} / e n u_{1}^{-}
$$

Independence of the electron concentration on film width is supported by the linear dependence of specific electrical resistivity $(\rho)$ on the inverse value of film width $(1 / d)$ in $77-110 \mathrm{~K}$ temperature range [8].

To analyze experimental data on galvanomagnetic phenomena in the bismuth films with 0.05 at. \% Te we 
will use the fact that the magnetoresistance value and consequently the carrier mobilities have a tenuous connection with temperature, in case carrier mobility is severely limited by film surface or structural defects, and the changes in specific electrical resistivity reflect temperature dependence of carrier concentration [8]. Length of free path of an electron in the bulk Bi crystal with 0.005 at. $\%$ Te is approximately equal to $300 \mathrm{~nm}$ under the temperature of $300 \mathrm{~K}$ [11].

To create conditions of significant limitation of carrier mobility by film surface and structural defects in the temperature range under study, a special Bi film with 0.05 at. $\%$ Te was produced, its width was far less than the length of free path in the bulk Bi crystal with 0.05 at. \% Te and equal to $60 \mathrm{~nm}$; then temperature dependence of the specific electrical resistivity of the film was measured. The experimental results reveal that the films that are less than $0.4 \mu \mathrm{m}$ at the temperature of 77 and $300 \mathrm{~K}(\rho(77) / \rho(300)$ have almost the same ratio of the specific electrical resistivity that shows significant limitation and weak temperature dependence of carrier mobility in these films [8].

The experiment resulted in deriving the dependence of specific electrical resistivity on temperature for 60$\mathrm{nm}$-wide film; so if we take into it into consideration and also assume that electrons and holes in the sample under research have approximately equal mobility that does not depend on the temperature, we shall find carrier concentration $n(T)-n(77)=p(T)$, that is determined by electron-hole pair excitation caused by increasing temperature, asserting that electron concentration $n(77)$ is the same as in bulk crystal $\left.((n) 77)=9,83 \cdot 10^{24} \mathrm{~m}^{3}, p(77)=0[11]\right)$ when the temperature is $77 \mathrm{~K}$. Hence:

$$
\begin{gathered}
p(T)=0,5 n(77) \cdot(\rho(77) / \rho(T)-1) \\
n(T)=n(77)+p(T)
\end{gathered}
$$

Employing formulae (5) and (6) and the experimentally obtained temperature dependence of specific electrical resistivity of $60-\mathrm{nm}$-wide Bi film with 0.05 at. \% Te, we calculated the temperature dependence of carrier concentration that was predetermined by electronhole pair excitation. The results of calculation are presented in [8].

Thus we proposed a model for carrier concentration calculation based on experimental data on the films for tellurium concentration that makes it possible to observe single-region approaching at nitrogen temperature.

The results of calculation of electrons and holes concentration within the framework of the proposed model agree well with the experimental data on the specific electrical resistivity and Hall coefficient for the block $\mathrm{Bi}(0.05$ at. \% Te) films that are less than $0.4 \mu \mathrm{m}$ wide in single region zones as well as in the temperature range that is characterized by electron-hole pair excitation.

We calculated temperature dependence of electrons and holes mobility for every Bi film with 0.050 at. \% Te, on the basis of the temperature dependence of the specific electrical resistivity and Hall coefficient, using expressions (1) and (3) and taking into consideration hole component and the obtained values of carrier concentration for the $60-\mathrm{nm}$-wide film and ratio $u_{1}^{-} / u_{2}^{-}$, calculated by means of expression (4) at $77 \mathrm{~K}$, and assuming independence of the $u_{1}^{-} / u_{2}^{-}$ratio on temperature and film width. The results are shown in Fig. 3-4.

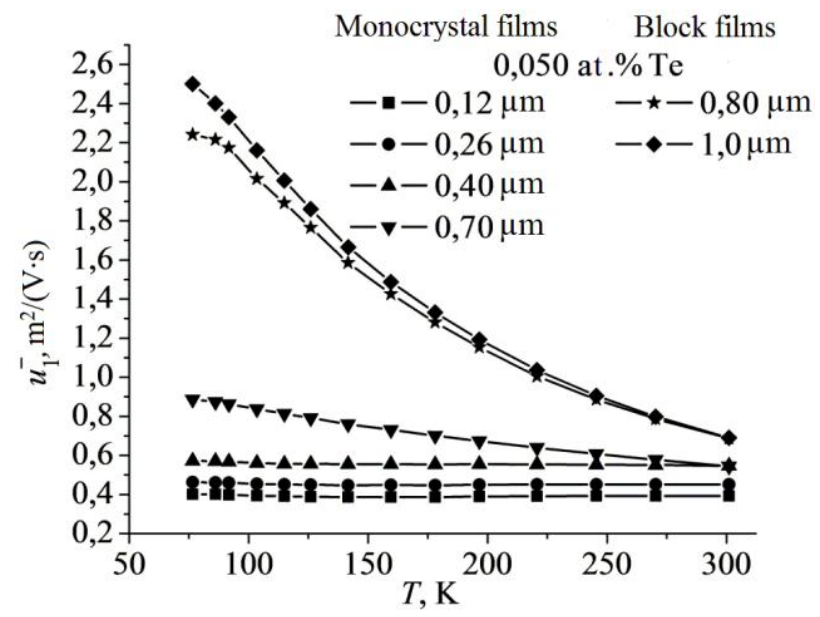

Fig. 3 - The temperature dependence of electron mobility in the block and monocrystal bismuth films doped with tellurium (tellurium contents -0.050 at. \% Te) that have different width

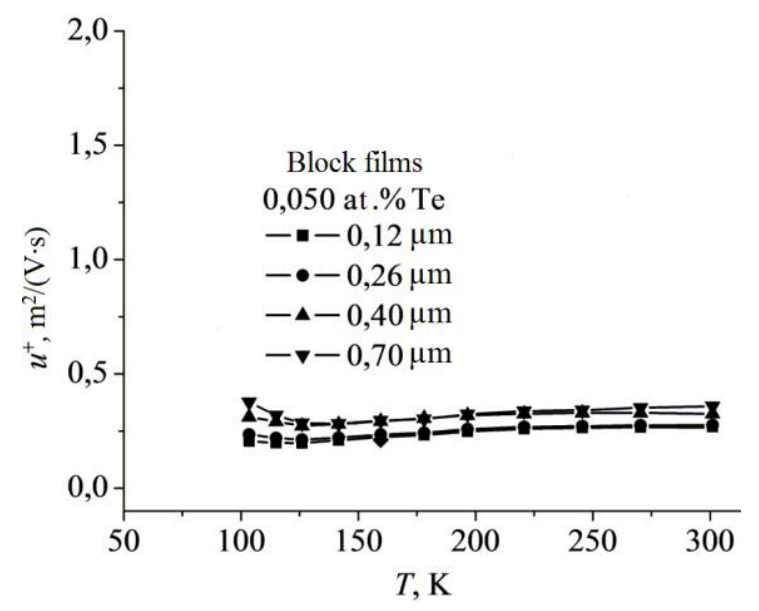

Fig. 4 - The temperature dependence of electron mobility in the block and monocrystal bismuth films doped with tellurium (tellurium contents -0.050 at. \% Te) that have different width

Fig. 3-4 give grounds to the conclusion that the values of electrons and holes mobility in the films that are less than $0.4 \mu \mathrm{m}$ have a tenuous dependence on temperature in case of significant limitation of carrier mobility due to film surface and structural defects.

The dependence of electrons $u_{1}^{-}$and holes $u^{+}$mobility on the width is most clearly observed in electrons (Fig. 3); it proves that film surface has a greater limiting effect on electrons than on holes. The electron mobilities in all temperature range have higher values.

Insignificant decrease of holes mobility in the low temperature range is connected with the considerable error of defining this mobility due to small concentration of the holes and their small contribution to the galvanomagnetic properties of the bismuth films doped with tellurium compared to electrons contribution.

Moreover, the temperature dependences on Fig. 1-3 
give grounds to conclude that increase of donor admixture of tellurium in the bismuth films results in decrease of electron mobility in the whole temperature range $[2,4,8]$.

\subsection{Matissen's Rule}

In case different scattering mechanisms [10] have an independent contribution to carrier mobility limitation by phonons $1 / u_{1 p h}^{-}$, surface $1 / u_{1 s}^{-}$, and defects that are present in the films (e.g. crystallites boundaries) and additional in relation to the bulk crystal $1 / u_{1 d e f}^{-}$, we can employ Matissen's Rule [8, 10]:

$$
1 / u_{1}^{-}=1 / u_{1 p h}^{-}+1 / u_{1 s}^{-}+1 / u_{1 d e f}^{-}
$$

To differentiate the various scattering mechanisms contribution to electron mobility limitation in the bismuth films doped with tellurium, dependences $1 / u_{1}^{-}=f(1 / d)$ were worked out for block and monocrystal films. The results for the temperature of $77 \mathrm{~K}$ are presented in Fig. 5.

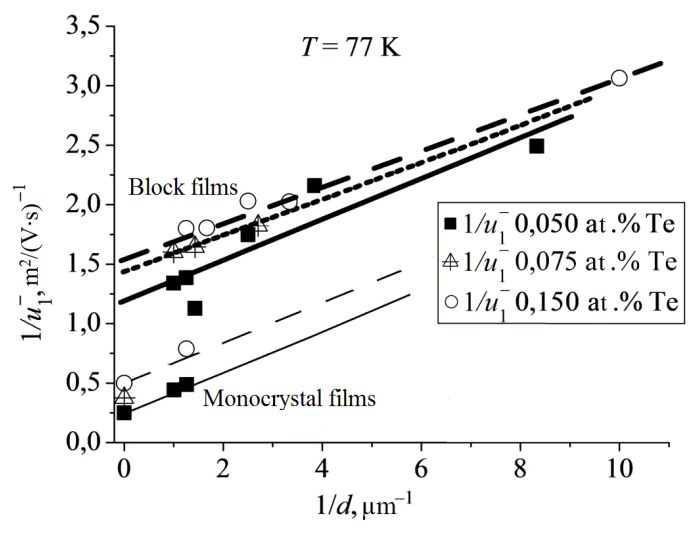

Fig. 5 - The dependence $1 / u_{1}^{-}$- on $1 / d$ in the block and monocrystal bismuth films with tellurium contents 0.050 at. $\%$, 0.75 at. $\%$ and 0.150 at. $\%$ at $77 \mathrm{~K}$. For $1 / d=0$ the values of $1 / u_{1}^{-}$of the bulk monocrystal are given

The same figure shows values of inverse electron mobilities in the bismuth monocrystals containing 0.050 at. \% Te, 0.075 at. \% Te, 0.150 at. \% Te and the monocrystal bismuth films containing 0.050 at. \% Te, 0.150 at. $\%$ Te.

As it can be seen in Fig. 5, the dependences
$1 / u_{1}^{-}=f(1 / d)$ for block films are close to linear. Extrapolation of these dependences at the given temperature to $1 / d \rightarrow 0$ yields values of carrier mobilities caused by scattering on phonons and additional film structural defects, i.e. values $1 / u_{1 p h}^{-}+1 / u_{1 d e f}^{-}[12]$.

The deduction of inverse mobility of the bulk bismuth monocrystal of the same composition $1 / u_{1 p h}^{-}$from this value defines the contribution of the film structural defects to the limitations of this mobility. Fig. 5 makes it obvious that this contribution does not depend on tellurium concentration and equals to $1 / u_{1 d e f}^{-} \approx 1\left(\mathrm{~m}^{2} / \mathrm{B} \cdot \mathrm{c}\right)^{-1}$, thus, has a correlation with the fact that increase of doping tellurium admixture concentration does not significantly change the size of crystallites in all the films under research.

In $0.80 \mu \mathrm{m}$-wide monocrystal films, the surface contribution $1 / u_{1 s}^{-}$also does not depend on doping degree and equals to: $1 / u_{1 s}^{-}=1 / u_{1}^{-}-1 / u_{1 p h}^{-} \approx 0,25\left(\mathrm{~m}^{2} / \mathrm{B} \cdot \mathrm{c}\right)^{-1}$.

These conclusions can be used in further analysis of the results for pure bismuth films and bismuth films with other concentrations taking into consideration scattering of electronic subsystem.

\section{CONCLUSION}

The proposed model of calculation of carrier concentration based on the experimental data for the 60-nmwide $\mathrm{Bi}$ film (0.050 at. \% Te) permits to define the values of electron and holes concentration in the electronhole excitation zones.

When contribution of phonon scattering decreases in the bismuth films doped with tellurium, classical size effect manifests itself in carrier mobility limitation that grows as the width of the films decreases.

In bismuth films with the contents of tellurium equal to 0.050-0.150 at. \% the contribution of film surface and structural defects to electron mobility limitation does not depend on tellurium concentration.

\section{ACKNOWLEDGEMENTS}

The author extends his gratitude to Vladimir Minovich Grabov, Doctor of Physical and Mathematical Sciences, Professor of General and Experimental Physics Chair of Herzen State University, Vladimir Alekseevich Komarov, Associate Professor who took an active part in the discussion of scientific results.

\section{REFERENCE}

1. V.M. Grabov, E.V. Demidov, V.A. Komarov, Solid State Phys. 50 No 7, 1312. (2008).

2. D.S. Orlova, E.I. Rogacheva, Nanosystems, nanomaterials, nanotechnologies. 7 No 2, 487 (2009).

3. J. Heremans, D.T. Morelli, D.L. Partin, C.H. Olk, C.M. Thrush, and T.A. Perry, Phys. Rev. B 38 (15), 10280 (1988).

4. W. Schnelle, U. Schnelle, phys. status solidi a 115 No 2,

505 (1989).

5. U. Dilner, W. Schnelle, phys. status solidi a 116 No 1, 337 (1989).

6. U. Dilner, W. Schnelle, phys. status solidi a 116 No 2, 715 (1989).

7. A.F Mayadas, M. Shatzkes, Phys. Rev. B. 1 (4), 1382 (1970).

8. V.M. Grabov, E.V. Demidov, V.A. Komarov, D. Yu. Mat- 
veev, A.A. Nikolaeva, D. Markushevs, E.V. Konstantinov, E.E. Konstantinova, Semiconductors 48 No 5, 630 (2014).

9. A.A. Ganeev, M.A. Kuzmenkov, S.V. Potapov, A.I. Drobyshev, M.V. Voronov, Mass Spectrometry. 3 No 3, 185 (2006).

10. V.M. Grabov, V.A. Komarov, E.V. Demidov, M.M. Klimantov, Herzen University Bull. 122, 22 (2010).
11. V.M. Grabov, V.A. Komarov, I.I. Khudyakova, T.A. Yakovleva, Physics of Semimetals and Low-Dimension Structures on their Basis: (SPb: Publishing house of Herzen State Pedagogical University: 2011)

12. H. Hattab, E. Zubkov, A. Bernhart, G. Jnawali, C. Bobisch, B. Krenzer, M Acet, R. Moller, M. Horn-von Hoegen, Thin Solid Films $\mathbf{5 1 6}$ No 23, 8227 (2008). 